EPJ Web of Conferences 66, 01006 (2014)

DOI: $10.1051 /$ epjconf/ 20146601006

(C) Owned by the authors, published by EDP Sciences, 2014

\title{
Probing Sea Quarks and Gluons: The Electron-Ion Collider Project
}

\author{
Tanja Horn ${ }^{1, a}$ \\ ${ }^{1}$ Catholic University of America, Washington, DC 20064, USA
}

\begin{abstract}
The 21st century holds great promise for reaching a new era for unlocking the mysteries of the structure of the atomic nucleus and the nucleons inside it governed by the theory of strong interactions (QCD). In particular, much remains to be learned about the dynamical basis of the structure of hadrons and nuclei in terms of the fundamental quarks and gluons. One of the main goals of existing and nearly completed facilities is to map out the spin flavor structure of the nucleons in the valence region. A future Electron-Ion Collider (EIC) would be the world's first polarized electron-proton collider, and the world's first $e-A$ collider, and would seek the QCD foundation of nucleons and nuclei in terms of the sea quarks and gluons, matching to these valence quark studies. The EIC will provide a versatile range of kinematics and beam polarization, as well as beam species, to allow for mapping the spin and spatial structure of the quark sea and gluons, to discover the collective effects of gluons in atomic nuclei, and to understand the emergence of hadronic matter from color charge.
\end{abstract}

\section{Introduction}

A fundamental goal of modern nuclear science is to discover, explore, and understand the fundamental constituents of matter, the hadrons and the nuclei, on the basis of the fundamental theory of strong interactions, Quantum ChromoDynamics (QCD) [1]. Strongly interacting hadrons and nuclei account for essentially all of the mass of the visible universe, and thus understanding their internal structure could be key to revealing the dynamical origin of mass and the behavior of matter at astrophysical temperatures and densities.

In the quest of understanding hadron structure in terms of the basic QCD degrees of freedom experiments involving electroweak probes are an important source of information. Extensive studies have mapped out the elastic form factors of the nucleon and revealed that nucleons viewed at smaller distance scales are themselves composed of pointlike constituents, called quarks. Subsequent experiments validated QCD and confirmed the existence of gluon degrees of freedom at small distance scales. For instance, experiments at high energies (small distance scales) measured the basic number densities of quarks and gluons in the nucleon. It is remarkable that one universal set of these quarkgluon distribution functions can interpret all high energy proton collision data above $2 \mathrm{GeV}$. As an example at low energies lattice QCD calculations of the light hadron masses are in good agreement with experimental data.

\footnotetext{
ae-mail: hornt@cua.edu
} 
Although much progress has been made on both experimental and theoretical fronts, several challenging mysteries of the strong force remain. For example, the three-dimensional structure of the nucleon is far from the simple valence structure it was initially thought to have. A central question is what role the non-valence called "sea" quarks and gluons play in nucleon structure, e.g., their spatial distributions, response to polarization, and the contribution of their orbital motion to the formation of nucleon spin. Another question is related to collective effects of gluons in nuclei, for instance, how the transverse spatial structure of gluons in the nucleon compares to that in the nucleon and the approach to a new regime of high gluon densities and saturation at high energies. Another question concerns the interaction of a strong color charge of QCD with nuclear matter, e.g., our understanding of the emergence of hadronic matter from colored quarks and gluons of QCD, and in particular, how nuclear matter responds to a fast moving color charge, and how the color charge evolves in space and time. Furthermore, our understanding of strong interaction dynamics, e.g., the conversion of energy into matter is still in its infancy.

A polarized $e-p / e-A$ collider (Electron-Ion Collider, EIC) with variable center of mass energy and polarized luminosity over the full energy range would be the ultimate machine to address these intellectually pressing questions related to our fundamental understanding of QCD. Such a facility would provide the required combination of kinematic reach, polarized luminosity, and detection capabilities to explore nucleon and nuclear structure well into the sea quark and gluon-dominated regime. The EIC would represent the natural extension of existing and nearly completed facilities, e.g., highluminosity, fixed target experiments (JLab $12 \mathrm{GeV}$, SLAC) and high energy electron-proton (unpolarized) colliders (HERA). In particular, polarized beams would allow for studies of proton and neutron spin structure with unprecedented accuracy and kinematic reach. The EIC would be the world's first $e$ - $A$ collider allowing for studies of QCD in the nuclear environment. The three main goals of the EIC are:

- Map the spin and spatial structure of quarks and gluons in nucleons and address questions like how much spin is carried by the gluons, what is the contribution of orbital motion of sea quarks and gluons, and what do partons reveal in transverse momentum and coordinate space.

- Discover the collective effects of gluons in atomic nuclei and address questions like if sea quarks and gluons are confined into individual nucleons in the nucleus

- Understand the emergence of hadronic matter from color charge, e.g., how does the nuclear medium respond to a fast moving color charge

\section{Science highlights}

\subsection{Nucleon Spin and 3D Structure}

\subsubsection{Spin and Flavor Structure}

The distribution of polarized gluons is an important measurement in solving the puzzle of the nucleon spin decomposition and quantifying the role of orbital angular momentum. Present data on polarized $p$ - $p$ collisions at RHIC essentially do not constrain the polarized gluon density for $x<\sim 0.05$. Inclusive measurements with an EIC would dramatically extend this data set and determine the polarized nucleon structure function $g_{1}$ down to $x=0.001$ with a substantial range in $Q^{2}$ allowing one to extract the polarized gluon density from the $Q^{2}$ dependence. The EIC is thus a powerful tool for revealing gluon helicity structure. In addition to the valence quarks, the nucleon contains a sea of quark and anti-quark pairs that is created by non-perturbative QCD interactions and reflects the complex structure of the ground state of the theory. The spin and flavor quantum numbers carried by the sea are 


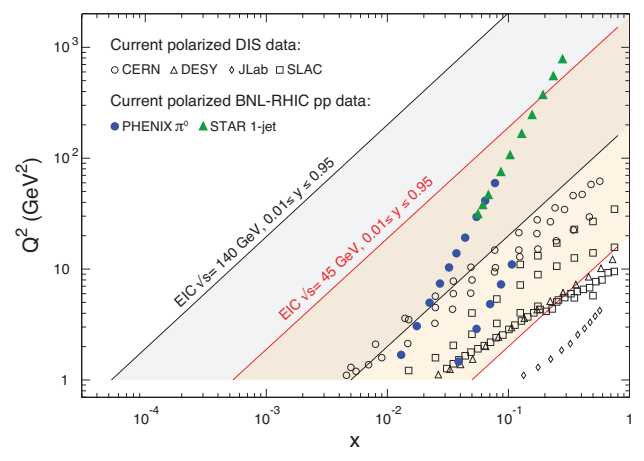

(a)

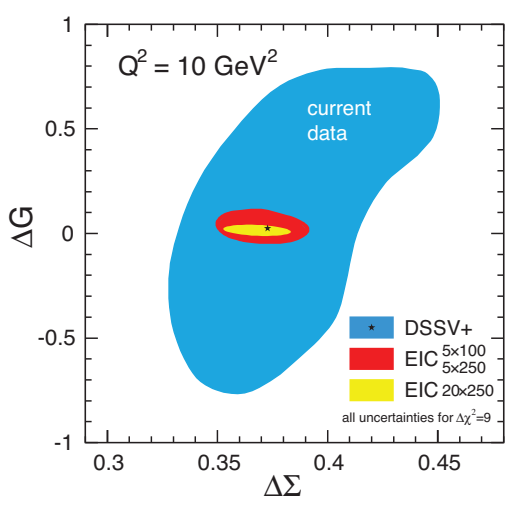

(b)

Figure 1. (a) Range in longitudinal parton momentum fraction $x$ vs. the square of momentum transferred by the electron to the nucleon $Q^{2}$ accessible with the EIC in $e-p$ collisions at two center of mass energies. (b) Present (outer area) and projected (inner area) accuracies for the correlated truncated integrals of quark $\Delta \Sigma$ and gluon $\Delta G$ helicity contribution to the proton spin over $0.001 \leq x \leq 1$ that would be achieved by the EIC for different center-of-mass energies. Figures from Ref. [2].

poorly constrained. The contribution of quark and anti-quark to the spin seems to be small. The EIC could measure the distribution of sea quarks through SIDIS. The kinematic coverage is uniquely suited for such measurements and will allow for a precise mapping of this largely unexplored component of the nucleon. Figure 1 shows the reduction in uncertainty of the contribution of the gluon, quark, and anti-quark spin to the nucleon in the range of $x=0.001$ to 1.0 .

\subsubsection{Confined motion of partons}

Transverse Momentum Dependent (TMDs) distributions are sensitive to quark/gluon and nucleon polarization, and correlations between them. They provide a three-dimensional representation of the nucleon in momentum space. TMDs can be accesssed through semi-inclusive scattering reactions in which the final state particles produced by the fragmentation of the struck quark or by nucleon fragments allow one to probe the quark and gluon transverse momentum and its correlation with the nucleon spin. There are eight independent TMD quark distributions, each of which describes a different aspect of nucleon structure, e.g., transverse motion and deformation, spin-orbit correlations, orbital angular momentum, or final-state interactions of the struck quark with the color fields in the nucleon. Measurements with the EIC will allow to precisely determine the valence and sea quark Sivers functions, which describes the transverse momentum distribution correlated with the transverse polarization vector of the nucleon. The Sivers function would be studied in a kinematic region, which has previously not been accessible and the region $x>0.01$ where it is expected to be sizeable. The EIC with good luminosity and high transverse polarization will be the optimal tool to reduce existing uncertainties and increase the kinematic reach. This allows for creating transverse momentum density distributions of, e.g., an up quarks moving with longitudinal momentum fraction in a transversely polarized proton moving in the $\mathrm{z}$ direction and polarized in the y direction. Achievable statistical precision of quark Sivers function obtained from EIC kinematics is shown in Figure 2. Currently no such picture exists for the gluon dominated regime in the proton. The EIC would be essential to initiate 
and realize such a program allowing to study all TMD distributions in detail over an unprecedented wide kinematic range.
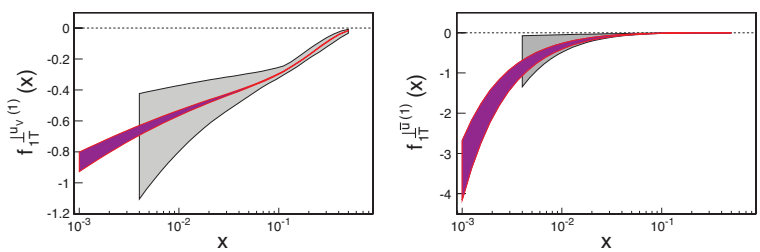

(a)

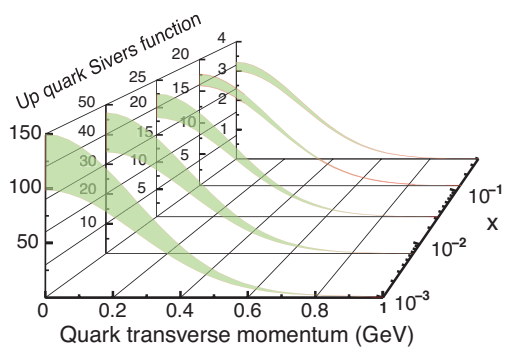

(b)

Figure 2. (a) Comparison of the precision of extractions of the Sivers function for the valence and sea quarks from currently available data (grey band) and from pseudo-data generated for the EIC with energy settings of $\sqrt{45}$ $\mathrm{GeV}$ (purple band). (b) Transverse-momentum profile of the up quark Sivers function at five $x$ values accessible to the EIC. Figures from Ref. [2].

\subsubsection{Spatial Imaging}

Closely related to the three-dimensional representation of the nucleon in momentum space is the question of the transverse spatial distributions of quarks and gluons carrying a longitudinal momentum fraction $x$. These distributions define the basic size and shape of the nucleon in QCD and convert the one-dimensional picture provided by longitudinal momentum densities into a full three-dimensional image of the fast-moving nucleon. The information on quark and gluon distributions in hadrons is provided by hard exclusive processes, in which a photon or meson originate from the struck parton and the nucleon remains intact. QCD factorization theorems allow for expressing amplitudes of such processes in terms of the Generalized Parton Distributions (GPDs), which combine the concept of quark/gluon distributions with that of elastic nucleon form factors. A large number of exclusive channels can be investigated at the EIC and each of them will give specific physics information. For example, measurements of $J / \Psi$ photo/electro production would be able to map the transverse spatial distribution of gluons, from a regime dominated by sea quarks and gluons to a region where valence quarks are important. This allows one to connect to the valence quark images expected from JLab $12 \mathrm{GeV}$ and COMPASS at CERN. Figure 3 shows the precision expected for the spatial distribution of gluons as measured in exclusive $J / \Psi$ production. This kinematic reach would also include the unexplored gluons in the valence region at $x>0.1$ whose presence has been inferred from global fits of deep inelastic scattering, but has not clearly been confirmed directly. The dynamical origin of these valence gluons is one of the outstanding key questions of nucleon structure in QCD. Information on the transverse spatial distribution of gluons is needed to describe the final states in $p p$ collisions at the LHC. Measurements of real photoproduction (DVCS) would allow one to differentiate gluon and quark spatial distributions and may reveal to what extent sea and valence quarks have different or similar characteristics. Production of light mesons with charge/isospin would map the transverse distribution of sea quarks. These measurements require differential measurements of low-rate processes with high precision, and thus rely on the high luminosity over the energy range envisioned for the EIC, and the possibility to longitudinally and transversally polarize the beam. 


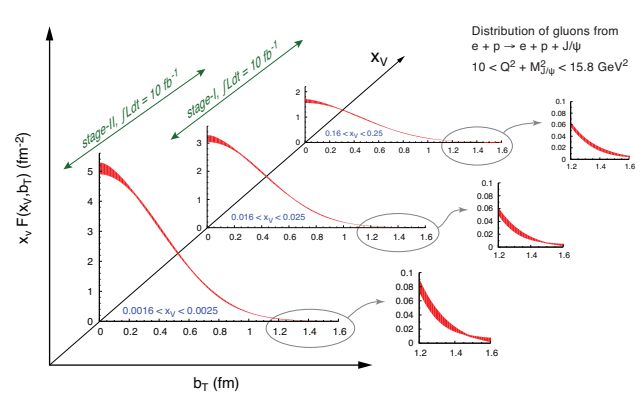

Figure 3. Projected precision of the transverse spatial distributions of gluons as obtained from the cross section of exclusive $J / \Psi$ production. Figure from Ref. [2].

\subsection{The Nucleus - a QCD laboratory}

\subsubsection{Quarks and Gluons in the nucleus}

A fundamental aspect of nuclear physics is to understand the structure and dynamics of QCD color fields in nuclei with nucleon number greater than unity. Experimentally this can be accomplished by scattering a small-size probe from nuclei over a range of incident energies. Earlier experiments have provided interesting information on the basic quark and gluon densities of nuclei. For valence quarks it was shown that in the fast moving nucleus their distribution is not a simple superposition, but measured ratio of structure functions following a non-trivial form of $x$. However, these experiments were unable to reach deep into the shadowing region distinguishing valence and sea quarks, or probe gluons. The EIC's wide kinematic coverage will allow for penetrating deeply into the shadowing region, while simultaneously having sufficient range to extract the nuclear densities through the $Q^{2}$ dependence of the structure function at both small and large $x$. Measurements at different energies would allow for isolating the longitudinal structure function, $F_{L}$, which provides direct access to gluons. Knowledge of the gluon distribution function would also be important in the search for the onset of gluon saturation.

Additional information on the nuclear modification of the quark/gluon structure of the nucleon may be obtained from deep-inelastic measurements, in which the spectator system of $A-1$ nucleons in the final state is detected. For example, scattering from the deuteron with a tagged spectator proton can measure the structure function of the bound neutron at controlled virtualities, from which the neutron quantities can be obtained by extrapolating to the on-shell point. In fixed-target experiments, scattering on bound neutrons is complicated. For instance, it is important to reach low momenta for true quasi-free reaction on neutron. The EIC is designed to tag spectators and all nuclear fragments and is thus well suited to provide completely new information on the bound proton structure functions and to explore the effects of embedding a nucleon in a complex nuclear environment.

\subsubsection{Propagation of color charges in nuclear matter}

The emergence of colorless hadrons from the colored quarks/gluons is a fundamental mechanism of QCD that still lacks a quantitative understanding from first principles. Basic questions concern the characteristic time scales for the neutralization of color charge and the formation of physical hadrons. Measuring these time scales would be the first step towards understanding how hadrons emerge dynamically from QCD color charges. Nuclear deep-inelastic scattering provides a known and stable 


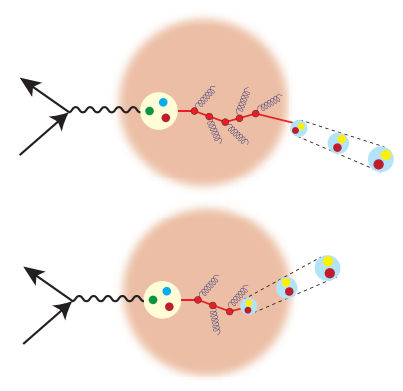

(a)

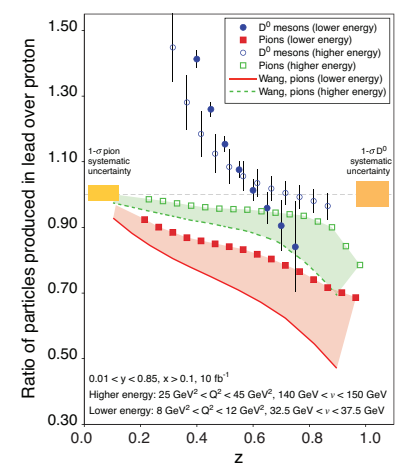

(b)

Figure 4. (a) Schematic for the interactions of the parton moving through cold nuclear matter when the prodiced hadron is formed outside (upper) and inside (lower) the nucleus. (b) The ratio of semi-inclusive cross sections for producing a single pion (red) and single $D^{0}$ (blue) in electron-lead collisions to the same produced in electrondeuteron collisions as a function of $z$ at the EIC with two different photon energies $v=35 \mathrm{GeV}$ at $Q^{2}=10 \mathrm{GeV}^{2}$ (closed symbols) and $v=145 \mathrm{GeV}$ at $Q^{2}=35 \mathrm{GeV}^{2}$ (open symbols). Figures from Ref. [2].

nuclear medium and a final state with good experimental control of the kinematics of the hard scattering. By measuring the energy loss and transverse momentum broadening of leading hadrons induced by the nuclear environment one can discriminate the different dynamical processes and infer evolution of the hadronization. The large $Q^{2}$ range permits measurements in the calculable perturbative regime with enough leverage to determine nuclear modifications in the QCD evolution of fragmentation functions. The large energy range allows one to experimentally boost the hadronization process in and out of the nuclear medium to cleanly extract the color neutralization and hadron formation times. The scattering and interactions between the nuclear medium and the produced partons can change the momentum distribution of the produced parton. The transverse momentum distribution broadens as a longer path is traversed and so can tell about how the quark/hadron interacted with the nuclear medium. Additional information on nuclei can be obtained by tagging nuclear fragments. As the quark propagates through the nuclear medium is interacts with nucleons and knocks them out either directly or as fragments. Subsequently the nucleus may be in an excited state and break up into other pieces. Those coming from direct interaction with the propagating quark will have higher momentum while those from breakup have lower momentum. Looking at the fast ones one can "follow" the interaction and study how many interactions there were on the way of the quark while $p_{T}$ broadening tells about the sum of interactions.

\subsection{EIC and its realization}

The EIC project is pursued jointly by BNL and JLab and both laboratories are working towards implementing a common set of goals. The first stage would have center of mass energies of $\sqrt{s}=20-70 \mathrm{GeV}$ and the second would have up to about $150 \mathrm{GeV}$ energies. Sometimes there are slight differences in emphasis based on site-unique choices for the solution. The BNL design would re-use the proton/ion beam of RHIC and collide it with an electron beam accelerated by energy-recovering linacs placed along a new recirculating ring in the same tunnels. JLab would re-use the $11 \mathrm{GeV}$ CEBAF electron accelerator and collide with ions from a newly built ion complex injected into a ring-ring $e-p / e-A$ 


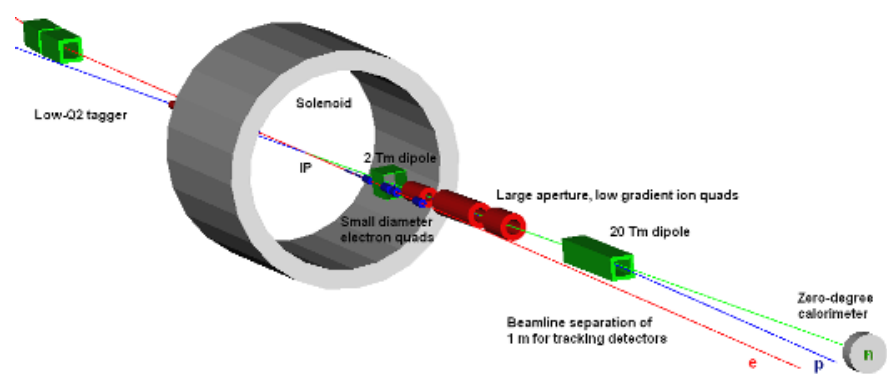

Figure 5. Forward ion detection in GEANT4 with a $50 \mathrm{mrad}$ crossing angle used for tracking simulations of the full-acceptance detector. Note that the final focusing quadrupoles are located $7 \mathrm{~m}$ from the IP, and the ZDC is on the outside of the ring. Figures from Ref. [2].

collider. The ring is laid out in the form of a Figure- 8 for optimal polarization transport and could support up to four interaction points.

The interaction region, acting as an interface between the beam acceleration/transport system and the detector, is one of the most challenging parts of the collider design. The high performance goals are achieved with a fully integrated detector and interaction region, which takes into account important physics constraints like beam transport and manipulation, single particle beam dynamics, collective effects and instabilities, detector acceptance, and synchroton radiation. To achieve full-acceptance, small angle detection is required on either side of the central detector. The low- $Q^{2}$ electron detection is easier to incorporate, while measuring forward and ultra-forward going hadronic or nuclear fragments along the ion direction is more challenging. In particular, ion fragments have a different rigidity from the beam, which means that they bend away from the beam and thus require large downstream apertures and small downstream magnet gradients to catch the particles. On the other hand, recoil baryons from, e.g., exclusive reactions, have a rigidity similar to the beam, i.e., stay in the beam, and thus require a small beam size at the second focus to get close to the beam and large dispersion to separate the scattered particles from the beam. Good momentum and angular resolution, as well as, sufficient separation between the beam lines are also important aspects.

At the EIC hadron detection will be done in three stages as illustrated in Figure 5. The first is the endcap with $50 \mathrm{mr}$ crossing angle. It covers all angles down to the acceptance of the forward spectrometer (several degrees around the ion beam line). The second element is a small $2 \mathrm{Tm}$ dipole magnet before the ion final focusing magnets to allow high resolution tracking of particles that do not enter the ion final focusing quadrupole (FFQ) magnets. This dipole augments the detector central solenoid at small angles where the tracking resolution would otherwise be poor. It covers angles to a few degrees, which is essential for providing wide coverage in $t$ for spatial imaging studies and to investigate target fragmentation. The FFQs have apertures sufficient for particles scattered at initial angles up to $20 \mathrm{mrad}$ in each direction for ion fragment with any rigidity. The third element is a 20 Tm large-acceptance dipole magnet a few meters downstream from of the FFQs to peel off spectator particles and allow for very small-angle detection (for particles passing the accelerator quadrupoles) with high resolution, which is essentially only limited by the intrinsic momentum spread of the beam. For a $50 \mathrm{GeV} / \mathrm{A}$ deuteron beam this provides a momentum resolution of better than $3 \times 10^{-4}$, i.e., 15 $\mathrm{MeV}$ resolution. This is an essential element for detecting recoil baryons and tagging of spectator protons in deuterium and other nuclear fragments. The design of this element is integrated with the accelerator and the $20 \mathrm{Tm}$ downstream dipole serves not only as a spectrometer, but also "corrects" 
the $50 \mathrm{mr}$ crossing angle and allows the neutrons to escape on a tangent to the ring, separating cleanly from the beam area before detection. This makes the electron and ion beam lines parallel in the $\sim 15$ $\mathrm{m}$ long drift space after the dipole, with separation of more then $1 \mathrm{~m}$, providing ample space for detectors. The optics give full angular acceptance for charged particles with up to $99.5 \%$ of the beam energy for all angles, and down to scattering angles of 2-3 mrad for all momenta. Detectors will be placed in front of the FFQs, between the FFQs and the $20 \mathrm{Tm}$ dipole, and in an extended, magnet-free drift space downstream of the latter. The apertures of the FFQs provide full neutron acceptance over $25 \mathrm{mrad}$ (total), centered close to zero. The neutrons (and boosted nuclear photons) will be detected in a zero degree calorimeter (ZDC) on the outside of the ring. In this configuration, any desired angular resoltion can be achieved by adjusting the distance of the ZDC (as well as its size). This results in an essentially $100 \%$ full-acceptance detector.

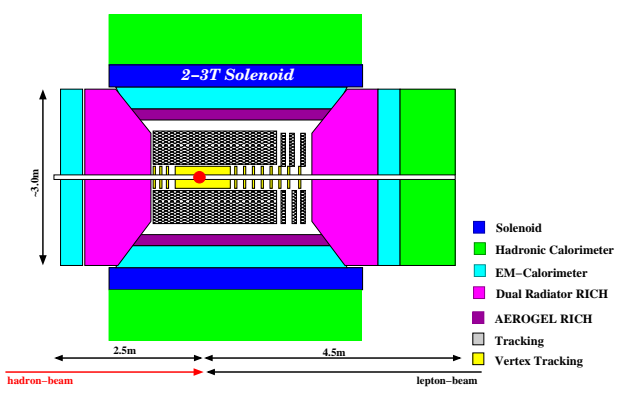

(a)

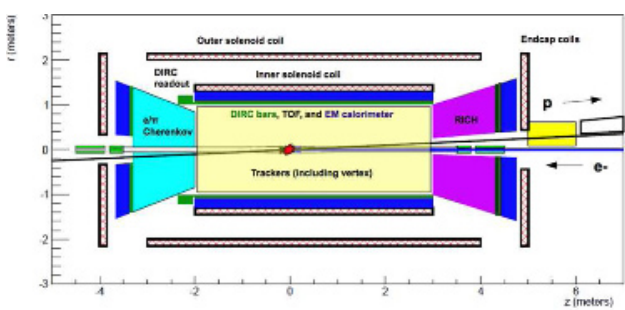

(b)

Figure 6. Schematic of the layout of the EIC central detector at (a) at BNL. (b) at JLab.

Though there are some minor differences in the layout of the detectors envisioned at JLab and BNL, driven in part by the space available in the IR, the general features and requirements are similar. To fullfill the requirements of hermicity the central detector will be built around a solenoid magnet. The constraints on the radial budget only allow for a limited number of options for the general layout. As illustrated in Figure 6 it would contain tracking, compact EM calorimetry and hadron PID provided by a compact ring imaging Cerenkov detector. The JLab schematic also explicitly includes a highprecision time of flight detector. Regarding the detector solenoid, the baseline solution is to use the solenoid from CLEO for one IP and the solenoid from BaBar for the other. However, substituting an iron-free dual-solenoid inspired by the 4th concept detector for ILC would offer many advantages, for instance, easier access, improved endcap acceptance, more compact endcaps due to active coils, and potentially lower mass and higher field. Details on the EIC project can be found in Refs. [2,3].

\section{References}

[1] DOE/NSF Nuclear Science Advisory Committee, "The Frontiers of Nuclear Science - A long Range Plan", 2007.

[2] EIC White Paper, "Electron Ion Collider: The Next QCD Frontier - Understanding the glue that binds us all", arXiv:1212.1701 (2012); IN10-3 report, arXiv:1108.1713 (2011).

[3] A. Abeyratne et al., "Science requirements and conceptual design for a polarized medium energy EIC at JLab", arXiv:1209:0757 (2012). 